**TITLE**

ASP Conference Series, Vol. **VOLUME ${ }^{* * *}$, **YEAR OF PUBLICATION**

**NAMES OF EDITORS**

\title{
Chandra and FUSE view of the WHIM: the Local Group and beyond
}

\author{
Fabrizio Nicastro \\ Harvard-Smithsonian Center for Astrophysics, 60 Garden Street, \\ MS-83, Cambridge, MA 02138, U.S.A.
}

\begin{abstract}
In this contribution, I review the current observational evidence for the existence of filaments of Warm-Hot Intergalactic Medium (WHIM). In particular, I first focus on the controversial issue of the identification of the $z \sim 0$ highly ionized far-ultraviolet (i.e. OVI) and X-ray (i.e. OVII, OVIII and NeIX) absorbers with either a very tenuous and diffuse WHIM filament, or with much denser condensations of material at large distances in the Galactic halo. I then present our recent detection (confidence level $>3 \sigma$ ) of the OVII WHIM at $z>0$ and derive an estimate of the total number of baryons contained in this hard-to-detect phase of the IGM.
\end{abstract}

\section{Introduction}

Despite recent progress in cosmology, very little is still known about the location of baryons in the local Universe. The extraordinary WMAP (Wilkinson Microwave Anisotropy Probe) measurements of the Cosmic Microwave Background $(\mathrm{CMB})$ anisotropies favor a $\Lambda$-CDM scenario in which the baryon density in the Universe amounts to $\Omega_{b}=(4.4 \pm 0.4) h_{70}^{-2} \%$ of the total matter-energy density, with a baryon-to-dark-matter ratio of $0.17 \pm 0.1$ (Bennet et al., 2003). This number agrees well with predictions by the standard 'big bang nucleosynthesis' when combined with light element ratios $\left(\Omega_{b}=(3.9 \pm 0.5) h_{70}^{-2} \%\right.$ : Burles \& Tytler, 1998), and also with the actual number of baryons at redshifts larger than 2 in the 'trees' of the Ly $\alpha$ Forest: $\Omega_{b} \geq 3.5 h_{70}^{-2} \%$ (Rauch, 1998; Weinberg et al., 1997). This concordance cosmology represents a major advance. However, the number of baryons detected in the "virialized Universe" at $z \sim 2$ (i.e. stars, neutral hydrogen associated with galaxies, and X-ray emitting gas in clusters) is more than a factor of 2 smaller than the concordance value, $\Omega_{b}<2 h_{70}^{-2} \%$

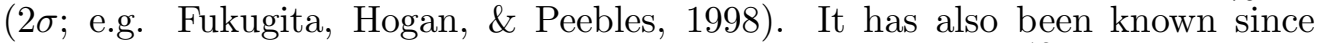
1959 (Kahn \& Woltjer, 1959) that, locally, more than $1.5 \times 10^{12} \mathrm{M}_{\odot}$ are needed to dynamically stabilize our own Local Group. So, one fundamental question arises: where have the baryons been hiding for the last $\sim 10$ Gyrs of life of the Universe?

Hydrodynamical simulations for the formation of structures in the Universe, predict that in the present epoch $(z \lesssim 1-2)$, half of the normal baryonic matter in the Universe (the "missing baryons") is in a tenuous (overdensities $\delta \simeq 5-50$, relative to the mean baryon density in the Universe) "warm phase" (the so called 

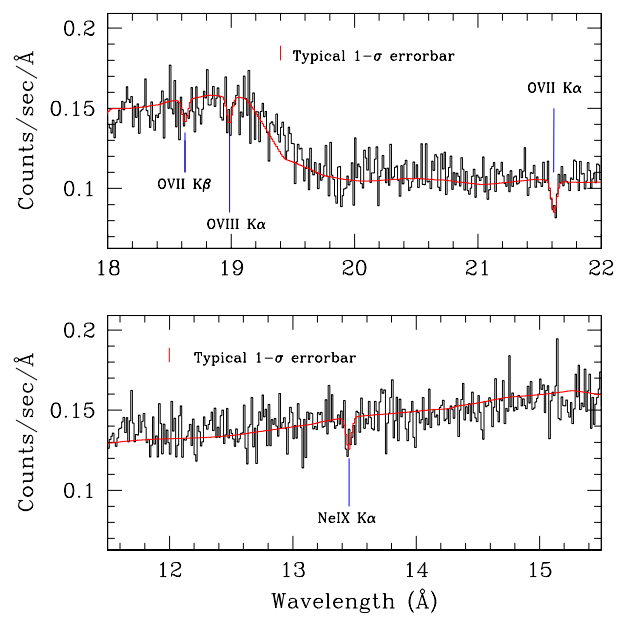

Figure 1. Two portions of the Chandra-LETG spectrum of PKS 2155-304. OVII $\mathrm{K} \alpha$ and NeIX K $\alpha$ resonant absorption lines at $z \sim 0$, from our Local Group WHIM filament, are clearly present at high significance, and associated OVII K $\beta$ and OVIII Ly $\alpha$ are detected at lower significance.

'Warm Hot Intergalactic Medium': WHIM), shock-heated to temperatures of $10^{5}-10^{7} \mathrm{~K}$ during the continuous process of structure formation (e.g. Hellsten et al., 1998; Cen, \& Ostriker, 1999; Davé et al., 2001). These hot filaments are now detectable with current instruments.

\section{The $z \sim 0$ Highly Ionized Absorbers: Local Group versus Galaxy Halo}

Two years ago we reported (Nicastro et al., 2002, hereinafter N02) the first detection of highly ionized $\mathrm{O}$ and Ne absorption $\left[\mathrm{OVII}_{K \alpha}(\lambda 21.602), \mathrm{OVIII}_{K \alpha}(\lambda 18.97)\right.$, $\left.\operatorname{NeIX}_{K \alpha}(\lambda 13.447)\right]$ at redshift consistent with zero, in the Chandra Low Energy Transmission Grating (LETG) spectrum of the blazar PKS 2155-304 (Fig 1). Detection of Li-like oxygen absorption $\left[\mathrm{OVI}_{2 s \rightarrow 2 p}(\lambda=1031.93,1037.62)\right]$ was also reported in the Far-Ultraviolet FUSE spectra of this (Sembach et al., 2000; Savage et al., 2000; N02) and many other lines of sight (Sembach et al., 2003: hereinafter S03; Savage et al., 2003; Nicastro et al., 2003: hereinafter N03). The resolution of the FUSE spectrometers is about 30 times better than that of the LETG, and clearly sufficient to resolve the OVI complex in at least two components: a high velocity (HV-OVI) component at $v_{L S R} \simeq-300$ to $-100 \mathrm{~km}$ $\mathrm{s}^{-1}$, and a narrower and more symmetric low velocity (LV-OVI) component at $v_{L S R} \simeq-30$ to $+100 \mathrm{~km} \mathrm{~s}^{-1}$ (see Fig. $3 \mathrm{a}$ in N02).

\subsection{A Controversial Solution}

Figure 2 shows that the UV absorbers (both, HV and LV) are incompatible with the X-ray absorbers in the hypothesis of purely collisional ionized gas with typical Interstellar Medium (ISM) densities (solid lines and superimposed $2 \sigma$ intervals for the ion abundance ratios). However, a single temperature solution 


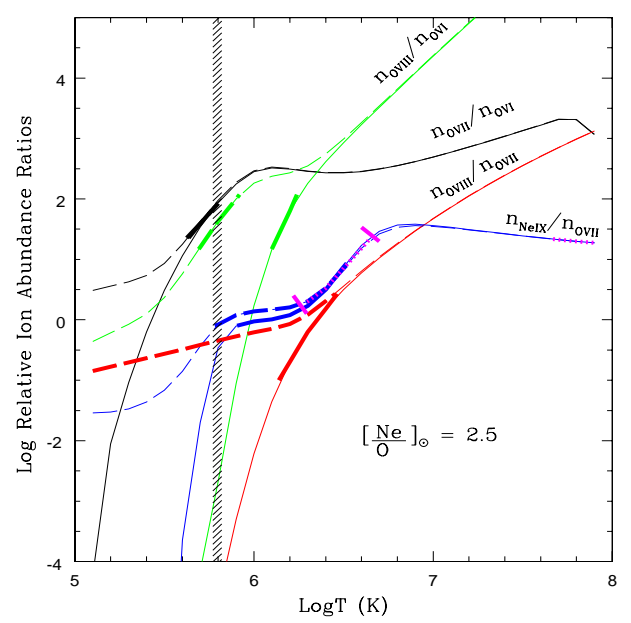

Figure 2. Expected ion abundance ratio curves for high-density gas in pure collisional ionization equilibrium (solid curve), and low density gas $\left(n_{e} \simeq 4-6 \times 10^{-6}\right.$ $\mathrm{cm}^{-3}$ : dashed curves). Thick segments on these curves are allowed $2 \sigma$ intervals for the measured EW line ratios.

(at $\mathrm{T} \sim 6.3 \times 10^{5} \mathrm{~K}$, dashed vertical line in Fig. 2) can be found for the HV-OVI and the X-ray absorbers if the volume density of the, mainly collisional ionized, gas is low enough (i.e. $n_{e}<10^{-5} \mathrm{~cm}^{-3}$ ) to allow photoionization by the extragalactic X-ray background to contribute (to $\sim 10 \%$ at $n_{b}=4 \times 10^{-6} \mathrm{~cm}^{-3}$ ) to the overall ionization balance in the gas. This is because the relative population of He-like and $\mathrm{H}$-like ions of $\mathrm{O}$ and $\mathrm{Ne}$, compared to Li-like $\mathrm{O}$, starts rising at relatively lower temperatures (i.e. $\mathrm{T} \sim 3-7 \times 10^{5} \mathrm{~K}$ ), compared to pure collisional ionization model (dashed lines and superimposed $2 \sigma$ intervals for the ion abundance ratios. See N02 for further details). This solution implies a baryon volume density of $n_{b}=4-6 \times 10^{-6} \mathrm{~cm}^{-3}$, corresponding to overdensities of $\delta=20-30$ relatively to the average baryon density in the Universe. Assuming homogeneity, and an $[\mathrm{H} / \mathrm{O}]$ ratio of 0.3 solar, this in turn gives a path-length of the absorber along this line of sight of $\sim 2-3 \mathrm{Mpc}$, so locating the absorber in the intergalactic space surrounding the Galaxy. Assuming a transverse size of this filament of $1 \mathrm{Mpc}$, gives a baryonic mass of $\sim(0.6-2) \times 10^{12} \mathrm{M}_{\odot}$. Inhomogeneity would imply a smaller size of the absorber, and so a lower mass. If, for example, the diffuse intergalactic medium would condensate when approaching the virialized structures of the Local Group, so mixing up with the ISM in the high galactic halo, up to densities of $n_{b} \sim 4 \times 10^{-4} \mathrm{~cm}^{-3}$, and if this layer of denser gas provided, say, $75 \%$ of the total observed equivalent baryon column, then the total filament would be about $1 \mathrm{Mpc}$ long along the line of sight to PKS 2155-304 (i.e. about the distance to M 31), with the dense shell contributing about $10 \%$ to the total mass of about $2 \times 10^{11} \mathrm{M}_{\odot}$.

The above solution (and consequent interpretation) is, of course, not unique. The alternative is that the X-ray and the ultraviolet absorbers are independently produced by a multiphase ISM (e.g. Heckman et al., 2002), or by both a hot multiphase ISM (the UV lines) and a diffuse local group medium or Galactic Corona (the bulk of the X-ray lines). The importance of this controversy lies in the total mass implied by the two different interpretations: in one case (Local 
Group WHIM: LG-WHIM) the implied mass is comparable, if not larger, than that of the most massive visible constituents of the Local Group, with strong implications for the Local Group dynamics and its stability, while in the Galactic scenario the gas mass would add up to a maximum of $\sim 1 / 100$ of the Galaxy's mass.

The Velocity Segregation of the HV-OVI One of the main pieces of evidence supporting the extragalactic solution (i.e. LG-WHIM solution), is the segregation of the HV-OVI in the Local Standard of Rest (LSR) frame. About 85 $\%$ of the lines of sight towards extragalactic sources observed by FUSE, show HV-OVI absorption (S03). Moreover, as demonstrated by N03, the majority of these absorbers appear to be at rest in the Local Group Standard of Rest (see Fig. 2 in N03). The velocity distribution of these absorbers is highly segregated in the Local Standard of Rest (LSR), with all negative-velocity absorbers lying at Galactic longitude $0^{\circ} \leq l<180^{\circ}$, and all positive-velocity absorbers lying in the opposite hemisphere (Fig. 2 in N03). This particular distribution is expected for objects at rest in the intergalactic space surrounding our Galaxy, with the kinematics in the LSR being an effect of the rotation of the Sun in the Galaxy's plane. This suggestion is confirmed by the progressive reduction of the degree of segregation (and so of the amplitude of the average velocity vector) of the HV-OVI absorbers for translations of the velocity vectors into the Galactic Standard of Frame (GSF) and the Local Group Standard of Frame (LGSR). In the LGSR the HV-OVI distribution appears random, and the amplitude of the average velocity vector is close to the FUSE resolution ${ }^{1}$. N03 concluded that the HV-OVI absorbers are at rest in the Local Group and so trace either a very extended Galactic Corona (Galactocentric distances $\gtrsim 150-200 \mathrm{kpc}$ ) or a LG-WHIM filament (see also Wakker, 2003, these proceedings).

S03 have argued against this interpretation. They noted that the above evidence is a necessary, but not sufficient condition to prove the LG-WHIM origin of the majority of the HV-OVI. While we certainly agree with this point, we want to stress here that their main argument against the LG-WHIM interpretation is the wide correspondence between some of the structures traced by the HV-OVI in the sky, and similar complexes seen in the hydrogen High Velocity Clouds (HVCs) distribution, and thought to be at distances of few kpc from the Galaxy center. However, both N03 and S03 were able to tentatively identify (based on very generous spatial and kinematics associations: up to tens of degrees in space and hundred $\mathrm{km} \mathrm{s}^{-1}$ in velocity) only about $18-20 \%$ of the HV-OVI with HVCs with known or estimated Galactocentric distance. These objects have not been considered in the statistical analysis presented by N03. For several other HV-OVI in their sample, S03 claim the association with known HVC structures (i.e. the Magellanic Stream, Complex C, Complex A), only on the base of the 'vicinity' (few degrees or tens of degrees apart) of these absorbers with the above HVCs structures.

\footnotetext{
${ }^{1}$ We stress that, unlike the case of the HI High Velocity Clouds, for the HV-OVI the amplitudes of the average velocity vector in the GSR and LGSR are inconsistent with each other at $>90$ $\%$ confidence level.
} 
Distribution of the Highly Ionized X-ray Absorbers Incredibly high quality Chandra and/or Newton-XMM grating spectra are needed to detect X-ray absorption lines with typical WHIM intensities (i.e. $\left.\mathrm{EW}\left(O V I I_{K \alpha}\right)<15 \mathrm{~m} \AA\right)$. The LETG spectrum of the blazar PKS 2155-304 (N02) was collected with an exposure of $60 \mathrm{ks}$, and during a powerful source outburst $\left(F_{0.5-2 \mathrm{keV}}=21 \mathrm{mCrab}\right.$ $\left.=4.2 \times 10^{-10} \mathrm{erg} \mathrm{s}^{-1} \mathrm{~cm}^{-2}\right)$. This guarranteed us about 700 counts per resolution element at the wavelength of the $\mathrm{OVII}_{K \alpha}$ line, enough to detect absorption lines with $\mathrm{EW}>10 \mathrm{~m} \AA$, at a significance larger than $3 \sigma$. Unfortunately, however, the flux level of PKS 2155-304 during this observation, was about 10-20 times that of the brightest nearby extragalactic sources in their quiescent levels. Very long exposures are then normally needed to obtain a sizeable signal-tonoise selected sample of X-ray spectra of extragalactic sources, suitable to study the distribution of the highly ionized X-ray absorbers at $z \simeq 0$. To date, only 5 extragalactic sources have such good quality spectra: PKS 2155-304, Mkn 421, 3C 273, NGC 4593 and NGC 5548. All of them show OVII $K \alpha$ absorption with similar intensities (i.e. about 10-20 mA: N02, Rassmussen et al., 2003, Fang et al., 2003).

Galactic HV-OVI or Ionized X-ray Absorbers Wanted Despite efforts to look for Galactic (or between the Galaxy and the two Magellanic Clouds) HV-OVI or highly ionized X-ray absorbers, none has been found yet.

We searched the literature for reports of, non-intrinsic, highly ionized (i.e. OVII, OVIII or NeIX) absorbers at LSR velocity consistent with $-500 \propto v_{L S R} \lesssim+$ $500 \mathrm{~km} \mathrm{~s}^{-1}$, in the X-ray grating spectra of binaries either in our Galaxy or in the two Magellanic Clouds, and could not find any. We found about a dozen published Chandra and/or Newton-XMM observations of X-ray binaries. In few cases variable (i.e. intrinsic) highly ionized emission and/or absorption, have been detected (e.g. Ness et al., 2003, ApJ, 594, L127). The remaining do not show any high ionization absoprption line either intrinsic or due to intervening ISM.

The situation is even clearer in the FUV. Zsargó, et al., (2003) report no detection of HV-OVI against any of the 22 O-B stars observed by FUSE in the Galactic halo. They also note that "if HV-OVI absorption was as common toward halo stars as toward extragalactic sources, one would expect 10-12 detections of HV-OVI absorption in" their "sample". They further note that this evidence "is very important because it justifies the association of the thick-disk OVI with the low-velocity gas". While we definitely agree with this conclusion, we also think that the above evidence directly suggests the association of the HV-OVI with extragalactic gas. Howk, Sembach \& Savage (2003) also report no detection of HV-OVI in the first $10 \mathrm{kpc}$, along the line of sight to the globular cluster M 3. Finally, only Galactic (i.e. Low-Velocity) OVI absorption, or OVI absorption at the velocity of the two Magellanic Clouds, is detected against samples of O-B stars in the two Clouds (Danforth et al., 2002; Hoopes et al., 2002). The few exceptions have Galactic explanation (i.e. a Supernova Remnant in our Galaxy, along the line of sight to AV 229).

Lower Ionization High Velocity Absorption One of the strongest arguments against the LG-WHIM interpretation for the HV-OVI and the X-ray absorbers, is the evidence that, along some lines of sight (i.e. PKS 2155-304, Mkn 509: Sem- 
bach et al., 1999), the HV-OVI have lower ionization counterparts (Si II/III/IV and C II/III/IV), with similar centroid velocity. This is not compatible with an homogeneous, diffuse and very tenuous WHIM surrounding our Galaxy, and producing the X-ray and the HV-OVI absorption. One possibility is therefore that the different absorptions are produced by a multiphase ISM, with typical ISM densities. However, inhomogeneities in the LG-WHIM could also easily give rise to a multiphase medium. The most efficient source of cooling in a line emitting plasma at $\mathrm{T} \sim 6 \times 10^{5} \mathrm{~K}$, is the $\mathrm{OVI}_{2 s \rightarrow 2 p}$ doublet. This mechanism is quite inefficient at typical WHIM densities, giving e-folding cooling times of 80 Gyrs, i.e. larger than the Hubble Time. Such a LG-WHIM obviously could not have had time to cool down to temperatures typical of the observed low-ionization species. However, an increase by a factor of 100 in density in compressed layers in proximity of the virialized structures of the Local Group, would reduce the cooling time to about 800 Myrs, about 2/25 the Galaxy's age. So, these denser layers of LG-WHIM, would have had time to cool down and produce the observed lower ionization species. Moreover, in the Local Group scenario, the velocity of the absorber in the LSR, is mainly due to our rotational motion in the Galaxy. So, along a given line of sight, any 'layer' (either Mpc- or kpc-thick) would produce absorption lines with roughly the same velocity in the LSR. The profile, however, would be different, depending mainly on the size of the absorber along the line of sight. This seems to agree with the profiles observed in low and high-ionization lines.

\section{The WHIM at $z>0$ : the Line of Sight to Mkn 421}

While evidence for the $z \sim 0$ filament is quite robust both in the FUV and in $\mathrm{X}$-rays ${ }^{2}$, until very recently there had been no clear detection in the X-rays of OVII-OVIII WHIM absorption at $z>0$ (either associated with the few known intervening OVI - Savage, Tripp, \& Lu, 1998; Tripp, Savage, \& Jenkins, 2000; Tripp \& Savage, 2000; Jenkins et al., 2003 - or faint - i.e. non-damped - H Ly $\alpha$ systems in the FUV, or at independent redshifts). The deepest observation of one of the brightest quasars at $z \sim 0.5$ (the $500 \mathrm{ks}$ LETG observation of $\mathrm{H} 1821+643)$ is sensitive only to $\mathrm{EW} \gtrsim 15 \mathrm{~m} \AA$ (at $\geq 3 \sigma$ level), and only $2-3$ low-significance $(\sim 2 \sigma)$ detections of such systems have actually been claimed (Mathur, Weinberg, \& Chen., 2003) along that line of sight, consistently with expectations.

This is mostly due to the intrinsic steepness of the 'LogN-Log(EW)' of the WHIM OVII-OVIII absorption lines, at relatively large EW (see, e.g., Fig. 4 in Hellsten et al., 1998). A single OVII K $\alpha$ system with $E W s \geq 18 \mathrm{~m} \AA$ is expected along a random line of sight, up to $z=0.5$. This number rises to 8 , for only twice weaker systems $(\mathrm{EW} \sim 9 \mathrm{~m} \AA$ ).

Very high signal to noise Chandra-LETG or Newton-XMM RGS spectra of extragalactic sources are therefore needed to detect the WHIM. These can

${ }^{2}$ Our Galaxy is embedded in the LG-WHIM and so, assuming spherical symmetry, any line of sight probes roughly about half the system and so sees large columns. The random orientation of intervening filaments, instead, greatly reduces the chances of observing such high columns. 
be provided with either multi-megasecond observations of a single line of sight, or observing Blazars when they undergo an outburst phase. We decided to pursue this second strategy, and during the past year triggered two Target-ofOpportunity Observations (TOOs) of the Blazar Mkn $421(z=0.03$, i.e. $d=128$ Mpc, De Vaucouleurs, et al., 1991), during two exceptionally high flux outburst phases. The two observations were carried out with the Chandra ACIS-LETG (2002, October 26-27) and the HRC-LETG (2003, July, 1-2), lasted 100 ks each, and reported $0.5-2 \mathrm{keV}$ fluxes of 100 and $40 \mathrm{mCrab}$, respectively. This allowed us to collect more than 10 million counts in the coadded first order spectrum, and more than 6000 counts per resolution element at $21.6 \AA$. In turn, this guaranteed a $3 \sigma$-sensitivity of $N_{O V I I} \gtrsim 10^{14.8} \mathrm{~cm}^{-2}$, more than an order of magnitude fainter than the LG-WHIM. Between the two Chandra ToO observations, Mkn 421 has also been observed by FUSE, following our request of a TOO.

Several absorption lines are clearly detected in the Chandra spectrum of Mkn 421 (Fig. 3a: left panel), from the relatively strong OVII K $\alpha$ line from the LG-WHIM at $\sim 21.6 \AA$ down to the OVII $\mathrm{K} \alpha$ line at the source redshift $(z=0.03)$. Our preliminary analysis suggests the identification of these lines with 3 different absorbers, one of which $\left(\lambda=21.852 \AA, N_{O V I I}=(1.2 \pm 0.3) \times 10^{15}\right.$ $\mathrm{cm}^{-2}$ ) a truly intervening WHIM system at $z=0.0116$, close ${ }^{3}$ to the redshift of a known weak Hydrogen Ly $\alpha$ system (Shull, Stocke, \& Penton, 1996). Upper limits $\left(\right.$ at $3 \sigma$ ) of $N_{O V I I I}<2 \times 10^{15} \mathrm{~cm}^{-2}$ and $N_{O V I}<2.2 \times 10^{13} \mathrm{~cm}^{-2}$ are also set on the OVIII K $\alpha$ (Fig. 3b: right panels) and $\mathrm{OVI}_{2 s \rightarrow 2 p}$ transitions from this system. Based on these measurements we estimate a temperature of the intervening WHIM OVII filament of $T=(0.7-2.5) \times 10^{6} \mathrm{~K}$, centered around the peak of the temperature distribution of the WHIM (e.g. Davé et al., 2001).

The number of OVII filaments per unit redshifts of $d N_{O V I I} / d z \simeq 35$, derived from this single detection, is in good agreement with expectations from hydrodynamical simulations (Hellsten et al., 1998). This is at least twice as much as the corresponding number for OVI $\left(d N_{O V I} / d z<14\right.$, Tripp, 2003, private communication), and so by far the largest reservoir of baryons in the local Universe.

Acknowledgments. F.N. acknowledges support by the Chandra grant GO2$3122 \mathrm{~A}$.

\section{References}

Bennett, C.L., et al., 2003, ApJS, 148, 1

Burles, S., \& Tytler, D., 1998, Space Sci Rev, 84, 65

Cen, R., \& Ostriker, J.P., 1999, ApJ, 514, 1

Danforth, C.W., et al., 2002, ApJ, 586, 1179

Davé, R., et al., 2001, ApJ, 552, 473

De Vaucouleurs, G., et al., 1991, "Third Ref. Cat. of Bright Galaxies", v.3.9

Fang, T., Sembach, K.R. \& Canizares, C.R., 2003, ApJ, 586, L49

\footnotetext{
${ }^{3}$ The H Ly $\alpha$ and the $\mathrm{OVII}_{K \alpha}$ systems are shifted in velocity by $420 \pm 120 \mathrm{~km} \mathrm{~s}^{-1}$, which supports the existence of multiphase WHIM.
} 


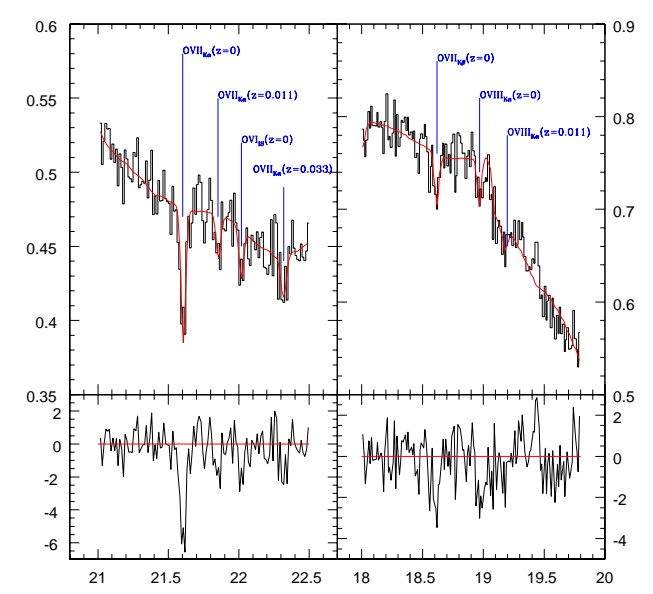

Figure 3. Two narrow portion of the coadded Chandra ACIS-LETG and HRCLETG spectra of Mkn 421, centered around (a) the OVII $K \alpha$ (left panels), and the OVIII $_{K \alpha}$ (right panels) transition. The solid continuous line in the bottom panels is the best fit polynomial plus absorption gaussians model to the data. Bottom panels show the residuals in $\sigma$ after the best fitting polynomial.

Fukugita, M., Hogan, C.J., Peebles, P.J.E., 1998, ApJ, 503, 518

Heckman, T.M., et al., 2002, ApJ, 579, 188

Hellsten, U., et al., 1998, ApJ, 509, 56

Hoopes, C.G., et al., 2002, ApJ, 569, 233

Howk, J.C., Sembach, K.R., \& Savage, B.D., 2003, ApJ, 586, 249

Jenkins, E.B., et al., 2003, AJ, 125, 2824

Kahn, F.D., \& Woltjer, L., 1959, ApJ, 130, 705

Mathur, S., Weinberg, D.H., Chen, X., 2003, ApJ, 582, 82

Nicastro, F., et al., 2002, ApJ, 573, 157: N02

Nicastro, F., et al., 2003, Nature, 421, 719: N03

Rasmussen, A., et al., 2003, HEAD, 35.0201

Rauch, M., 1998, ARA\&A, 36, 267

Savage, B.D., Tripp, T.M., Lu, M., 1998, AJ, 115, 436

Savage, B.D., et al., 2000, ApJ, 538, L27

Savage, B.D., et al., 2003, ApJS, 146, 125

Sembach, K.R., et al., 2000, ApJ, 538, L31

Sembach, K.R., et al., 2003, ApJS, 146, 165: S03

Shull, J.M., Stocke, J.T., \& Penton, S., 1996, AJ, 111, 72

Tripp, T.M., Savage, B.D., \& Jenkins, E.B., 2000, ApJ, 534, L1

Tripp, T.M., \& Savage, B.D., 2000, ApJ, 542, 42

Weinberg, D., et al., 1997, ApJ, 490, 564

Zsargó, et al., 2003, ApJ, 586, 1019 